\title{
A Study on the Adaptive Tabu Algorithm for the Dynamic Vehicle Routing Problem
}

\author{
Hsien-ming Chiu ${ }^{1}$ \\ ${ }^{1}$ Department of Transportation Management, Tamkang University
}

\begin{abstract}
In this paper, a five-phase solution procedure based on the Tabu search is proposed to solve the dynamic vehicle routing problem with real time demand information. A series of case studies with different characteristics such as demand density, demand size are used to test the solution capability of the proposed algorithm.
\end{abstract}

Keywords: Dynamic Vehicle Routing, Heuristic algorithm and Tabu Search.

\section{Introduction}

With the rise of electronic commercial, more and more firms have devoted themselves on the application of this type commerce in their business operation. With the availability of the real time demand and traffic information from the Electronic commerce and Intelligent Transportation System, a delivery operation planar need to develop a state-of-the-art control mechanism in order to provide a quick response delivery operation update to deal changes in these input information. In this paper, a five-phase solution procedure based on the Tabu search was proposed to solve the dynamic vehicle routing problem with real time demand information.

\section{Problem statement}

The problem addressed in this study is defined as follow: The service area of the delivery operation is set as $\mathrm{G}=(\mathrm{V}, \mathrm{E})$, where $V=\left\{v_{0}, v_{1}, \ldots \ldots, v_{n}\right\}$ is the set of nodes and, $E=\left\{\left(v_{i}, v_{j}\right): i<j\right\}$ is the set of arcs. In this service area, the depot is represented by $v_{0}$, and customers are represented by other nodes. The service time window for each customer is set to be $\left[e_{i}, l_{i}\right]$, the distance between two customer is symmetric and represented by a matrix $\left\{D=\left(d_{i j}\right)\right\}$. Under the assumption that the locations of customers unchanged, the changes in demand requirements are limited to the quantity of demand requirement for each customer. If the current cargo can not satisfy the new demand requirements, then traffic manager may have to introduce new vehicle and change the route structure such as to meet all the service requirements with minimal the total delivery cost.

Due to the dynamic nature of demand change, this type of problem should be treated as a dynamic vehicle routing problem. In this study, we adopt a dynamic approach to update the route structure such as to provide quick response to demand changes. If the new demand can not be met by the existing cargo, then it will be served by other vehicles. With real time information and communication, we can re-optimize all the remaining delivery operation to meet all the service requirements with minimum total cost.

The structure of this problem can be summarized as follow:

Inputs: network, demand, and vehicle data

Objective: minimum total delivery cost

Constraints: service and network limitations.

Outputs: set of routes and optimal fleet size.

\section{Model}

The notations used in this study are as follows:

$\mathrm{N}=$ Set of nodes

$\mathrm{R}=$ Set of routes

$\mathrm{M}=$ Set of vehicles

$\mathrm{Q}=$ Vehicle capacity

$T=$ Daily maximum work hour

$F=$ Fixed cost of a vehicle

$\alpha_{i j}=$ Unit penalty cost of unsatisfied demand

$x_{i j m}=\left\{\begin{array}{l}1, \text { If point } \mathrm{i} \text { to point } \mathrm{j} \text { served by vehicle } \\ \mathrm{m} .\end{array}\right.$ 0 , otherwise

$y_{i j}=\left\{\begin{array}{l}1, \text { if delivery is failure } \\ 0, \text { otherwise }\end{array}\right.$

$D_{m}=\left\{\begin{array}{l}1, \text { vehicle } m \text { is used } \\ 0, \text { otherwise }\end{array}\right.$ 
$q_{i}=$ Demand requirement in point $\mathrm{i}$.

$c_{i j}=$ Travel cost from point $\mathrm{i}$ to point $\mathrm{j}$.

$e_{i}=$ Starting time of the time window for point $\mathrm{i}$.

$p_{e}=$ Penalty cost of starting time window violation.

$l_{i}=$ Closing time of the time window for point $\mathrm{i}$.

$p_{l}=$ Penalty cost of closing time window violation.

$b_{i}=$ Delivery arrival time of point $\mathrm{i}$.

$S_{i}=$ Service time of point $\mathrm{i}$.

$t_{i j}=$ Travel time from points $\mathrm{i}$ to $\mathrm{j}$.

$P_{i}\left(b_{i}\right)=$ Penalty cost for point $\mathrm{i}$ at time $b_{i}$.

The mathematical model used in this study is as follow:

$\operatorname{Min} \sum_{i \in N} \sum_{\in \in N} \sum_{m \in M} c_{i j} x_{i j m}+\sum_{m \in M} F D_{m}+\sum_{i \in N} P_{i}\left(b_{i}\right)+\alpha \sum_{i \in \mathbb{N}} \sum_{\in \in \mathbb{N}} y_{i j}\left[\left(\sum_{i} q_{i}\right)-Q\right]^{(3.1)}$

subject to

$\sum_{i \in N^{o}} x_{i p}-\sum_{j \in N} x_{p j}=0, \forall p \in N$

$\sum_{i \in N} \sum_{j \in N} x_{i j m}\left(s_{i}+t_{i j}\right) \leq T, \forall m$

$\sum_{i, j \in S} x_{i j m}=|S|-1$, for all $S \subseteq N$

$x_{i j m} \in\{0,1\}, \forall(i, j) \in N, \forall m \in M$

$y_{i j} \in\{0,1\}, \forall(i, j) \in N$

$P_{i}\left(b_{i}\right)=\left\{\begin{array}{lcc}f_{a}+p_{e}\left(b_{i}-e_{i}\right)^{2}, & \text { if } \quad b_{i}<e_{i} \\ 0 \quad, & \text { if } \quad e_{i} \leq b_{i} \leq l_{i} \\ f_{b}+p_{l}\left(l_{i}-b_{i}\right)^{2}, & \text { if } \quad l_{i}<b_{i}\end{array}\right.$

$D_{m} \in\{0,1\}, \forall m \in M$

The objective function in this model represents the sum of vehicle travel cost, fixed cost, time window violation cost and penalty cost for unsatisfied demand. Flow continuation is guarantee by Constraint (3.2). Constraint (3.3) makes sure the maximum work hour limitation is kept. Subtour breaking constraint is shown as constraint (3.4). Constraint (3.5) is the penalty cost function for time window violation. The remaining constraints are the definition of the decision variables.

\section{Solution procedure}

There are two major components in the proposed solution procedure, i.e., route formation and route reconstruction. With real time update mechanism in the procedure, the real time demand information is fed into the solution procedure periodically, which may initiate the route reconstruction in order to provide a quick response to the demand change.

The solution procedure begins with the construction of initial route structure based on the preset demand requirements of the existing customers. If there is new demand request to delivery center which will exceed the service capacity of the current vehicle assignment, then we define this scenario as routing failure. Once there is routing failure, some vehicles will be reassigning back to depot for cargo refilling. However, in this study, this scenario may also be dealt with the reconstruction of remaining route structure based on the real time demand requirements, vehicle tracing and current service capability. This may provide a more flexible response to demand change without forcing vehicle back to depot which maybe more costly.

If there is a routing failure, then the routing reconstruction procedure is preceded. In the solution procedure proposed in this paper, the timing for the checking of the occurrence of the routing failure will be taken place with fixed time interval. All the real time information, such as unserved demand requirements, location and remaining cargo of each vehicle are reviewed in each checking time. Based on the real time information, the traffic manager can determine whether the routing failure is occurred. Once there is routing failure, the route structure is then reconstruction based on this information. In each checking time, we evaluate the possibility of the remaining cargo of each vehicle to meet the unserved demand requirements in current route structure. Once there is unsatisfied demand, we will mark these customers as unassigned customers for the service of other vehicles in the reconstruction of route structure. It is quite possible that a vehicle may be in the midway to serve an assigned customer in the checking time, which will make the situation more complex to deal with. To reduce the complexity of the solution procedure, these proceeding services will be treated as the demand to be served in the route reconstruction. Their service will be carried on first, and treated as demand served.

There are five major control points in the proposed solution procedure, i.e., the violation check of the maximum allowable working hour, the satisfaction of all the demand requirements, the reach of the checking time, the arrival of the new demand requirements and the assessment of routing failure. The first control point is used to evaluate the violation of the maximum 
allowable work hour, if it occurs, the procedure is then terminated, which means there is unserved demand in the solution. The evaluation of the accomplishment of all the demand requirements is conducted in the second control point. If all the demand requirements are served then the solution is completed. Otherwise, the route reconstruction will be proceeding.

The termination of the solution procedure is performed by the above two control points. The third control point is used to determine if it the time for the real time information update. If it is time to update the real time information, the fourth control point is then used to determine whether the next step should be conducted. If there is no new demand requirement then nothing needs to be done. Otherwise, the input of new demand requirements for the delivery operation will initiate the assessment of routing failure, which is the fifth control point of the proposed procedure. If the inclusion of new demand requirement will not cause routing failure, nothing needs to be done. Otherwise, the route reconstruction is conducted to meet new demand requirements with minimal total delivery cost.

These five control points are the major steps of the proposed solution procedure. With this procedure, not only the dynamic demand requirements can be dealt in the process, but also the route structure is update adaptively in order to reduce the possibility of routing failure with minimal total delivery cost.

There are two major components of the proposed solution procedure deal with the route structure, i.e., initial route structure and route structure reconstruction. There are significant efforts devoted in the solution algorithms of the classic vehicle routing problem for the past twenty years. A series of meta-heuristic procedure were proposed for this type of problem. In this study, a Tabu search algorithm is selected as the basis of the initial routing procedure in the proposed solution procedure. The Tabu search algorithm was proposed by Glover [1], and has quickly become one of the widespread meta-heuristic methods for the combinatorial problem. A series of studies have devoted their efforts on the application of the Tabu search in the classic vehicle routing problem with significant success. Several studies addressed their application on the stochastic vehicle routing problem with significant success too.

The use of the Tabu list in the search algorithm would result in an effective search such as to avoid the cyclic search. In the search process, an effective memory system is adopted to store the previous search history. This information is useful in searching for a better solution based on the lessons form previous experiences. With a sequential route structure revised procedures incorporated into the process to deal with the case of routing failure, the impact of this type of memory technique on the solution quality improvement is more significant in this study than other techniques.

If routing failure is occurred, the route structure reconstruction procedure is then conducted. Under this situation, the unserved demand requirements are marked, which will be served by the newly assigned vehicles without any violation of the existing constraints. The newly formed routes are then included in the set of delivery routes. This set of routes is treated as the new initial solution which will be improved by the route swap procedure in the solution procedure in order to reduce total delivery operation cost. With the sequential delivery routes reconstruction, the solution of the previous stage is treated as the initial solution for the current stage, which would improve the search speed and the quality of the solution. When all the demand requirements are met or the maximum allowable work hour is reached, the solution procedure terminated, and the final set of delivery routes and fleet size are the outputs of this procedure.

To keep tracing of the dynamic data in the proposed solution procedure, six arrays are used in the process. The purposes of these arrays are discussed as follows: First, travel time data array is used to record the travel time/distance between any two points in the service area. The next visiting point, distance to that point, and remaining cargo for each delivery vehicle is stored in the vehicle attribute array. The current un-served demand requirements are shown in the demand array. The vehicle routing array records all the route structure of the current set of delivery routes. The time window data is stored in the time window array. The service time for each point is recorded in the service time array. With these six arrays, all the data and solution information can be stored and retrieved in each steps of the solution procedure.

The basic characteristics of the Tabu search include the initial solution construction, the definition of neighborhood, the design of Tabu list and the assessment of the solution update. The search procedure begins with the initial solution selection as the starting point of the search process. In the following search process, solutions possessing some attributes of some recently visited solution are declared forbidden or Tabu to avoid cyclic search. A Tabu list is set to record these forbidden solutions. The design of the structure and length of this list have significant impact on the decrease of the likelihood of being trapped into a local optimal solution. But this list may limit the search area of the process, which can be overcome by the introduction of the aspiration principle.

The solution procedure of the Tabu search algorithm can be summarized as follows: 
Step 1: Find an initial solution from current feasible solution and denoted it as $\mathrm{X}_{\mathrm{o}}$. In addition, it also defines this solution as the current solution $\mathrm{X}$. Let this solution also as the current best solution, i.e., $X^{*}=X_{0}$. Set Tabu list as empty.

Step 2: From the neighborhood of current solution $X$, identify the best neighborhood solution $X^{\prime}$.

Step 3: If the move from $X$ to $X^{\prime}$ is in the Tabu list, go to step 5, otherwise go to step 4 .

Step 4: Record the move into the Tabu list, if $X^{\prime}$ better than $X^{*}$, then $X^{*}=X^{\prime}$, go so step2.

Step 5: If the move is within the aspiration principle, then relieve it from the Tabu list, make the move, and go to step 2. Otherwise, go to step 6.

Step 6: If the stopping rule is satisfied, then the process terminates, otherwise, set the current best feasible solution as the current solution $\mathrm{X}$, update Tabu list and go to step 2 .

The initial solution of the first step is derived from the nearest insertion, which had been proven quite effective. The neighborhood solution is derived from the 2-swap exchange of the two nodes in different routes. To increase the speed of the process, the best movement of partial set of potential movements is selected as the move to the neighborhood such as to reduce the search time for each new movement.

The Tabu list used in this process is represented by an $\mathrm{n} x \mathrm{n}$ array, with $\mathrm{n}$ denoted the number of nodes in the service area. The step of the move is recorded in this array. Based on the previous study, the length of 7 would provide an effective search process in the Tabu search algorithm.

For any move $\mathrm{m}$, if a better solution can be reached, then the move should be accepted. To prevent a better solution is falling in to the Tabu list, we need set a threshold such that if the improvement of a move is exceed this level, we will relieve if from the Tabu list such that this desirable movement is accepted, which is defined as aspiration principle.

The stopping rule of the process used in the paper is the limitation on the generation of the un-improvement solution. Although the quality of the solution may be improved with an increase in this limitation, but the computation time would be increased in the same time, which may not be justified. Based on the review of the previous studies, the number of 20 is used as the limitation on the generation of the un-improvement solution.

In the numerical experience, we first used a small size case to test and validate the proposed solution procedure and coding. The proposed Tabu search algorithm demonstrates its capability to construct an effective route structure for different sizes of problem. In this numerical testing, all the coding are written in Borland C language and executed in Pentium III PC.
The 50 points case of the Cristifofield and Eilon [2] was selected as the basic case study in the validation test of the proposed procedure. The dynamic demand requirements are then simulated with a random generator based on preset distribution pattern. Three types of distribution functions, i.e., Uniform distribution, Normal distribution, and Poisson distribution are used in the simulation process. The optimal fleet sizes derived from the proposed solution procedure under each distribution pattern with different confidence intervals are shown in Table 1.

\begin{tabular}{lllll}
\hline \multirow{2}{*}{$\begin{array}{l}\text { Distribution } \\
\text { Pattern }\end{array}$} & \multicolumn{4}{l}{ Optimal number of vehicle } \\
\cline { 2 - 5 } & $\geq 80 \%$ & $\geq 90 \% \geq 95 \%$ & $\geq 97.5 \%$ \\
\hline Uniform & 15 & 15 & 16 & 16 \\
Normal & 14 & 15 & 15 & 15 \\
Poisson & 16 & 17 & 17 & 17 \\
\hline
\end{tabular}

Table 1 Optimal fleet sizes under different confidence interval and distribution pattern.

\section{Concluding Remarks}

In this study, a five-phase solution approach based on the Tabu search is proposed to solve stochastic vehicle routing problem with real time demand information. A dynamic routing assignment procedure is developed to reach robust optimization of the delivery operation, which should provide the competition edge for the firm under consideration. A series of case studies with different characteristics such as demand density, demand size are used to test the solution capability of the proposed procedure. The numerical results demonstrated the capability of the proposed solution procedure in dealing with the dynamic demand requirement.

\section{Acknowledgement}

The author wishes to express his gratitude to the National Science Committee for their fund supporting.

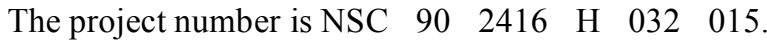

\section{References}

[1] Glover, F. 'Future Paths for Integer Programming and Links to Artificial Intelligence'. Computers and Operations Research, 13, 533-549, 1986.

[2] Christofides, N. and Eilon, S., "An Algorithm for Vehicle Dispatching Problem." Operational Research Quarterly 20, 309-318, 1969. 